\title{
IV Thrombolysis in Stroke From a Cavernous Carotid Aneurysm to Artery Embolus
}

\author{
Jamsheed A. Desai, Albert Yongwon Jin, Michel Melanson
}

Can J Neurol Sci. 2011; 38: 352-353

\section{CASE}

A 54-year-old man with a history of known right carotid cavernous aneurysm, migraine headaches, and a 20 pack per year smoking history presented two hours after acute onset of left hemiparesis. The patient had no other vascular risk factors and was not on any prescription medication. Initial National Institutes of Health Stroke Scale (NIHSS) score was 9 with examination showing a right gaze preference, left face and arm $>$ leg weakness, dysarthria and left inattention. Additionally an acute onset partial right Oculomotor and Abducens palsy accompanied by diplopia was appreciated. Computed tomogram (CT) head visualized a) hypoattenuation within the right lentiform nucleus with an Alberta Stroke Program Early CT (ASPECT) score of 9 and b) bilateral prominence of the cavernous sinuses (Figure A). The CT angiogram revealed an eccentric mural thrombus (Figure B) occupying approximately $50 \%$ of a $16 \mathrm{~mm}$ (diameter) right fusiform carotid cavernous aneurysm (CCA) (Figure D). No proximal large vessel disease or cardiac rhythm disturbance was identified. The CT perfusion imaging (not shown) highlighted a blood volume defect centered within the right lentiform nucleus as well as blood volume defect extending to cortical layers of the majority of the right middle cerebral artery territory, with corresponding blood flow and mean transit time deficits. After discussion with patient and his family, intravenous recombinant tissue plasminogen activator (rtPA) was administered. Factors which were considered in arriving at his decision included: the potential to salvage a large territory at risk, a high ASPECT score indicating relatively preserved parenchyma at the time of presentation, severity of his neurologic deficits, young age of the patient, and the relatively low probability of rupture of cavernous carotid aneurysms. Follow-up diffusion weighted magnetic resonance imaging (MRI) demonstrated right middle carotid artery (MCA) lenticulostriate branch territory infarction (Figure C) representing near complete salvage of the threatened right MCA territory. Follow-up examination showed resolution of the partial right IIIrd and VIth nerve palsy, likely due to resolution of mass effect caused by acute thromboses of the right CCA. The patient made a near complete motor recovery with residual mild left arm weakness on discharge, with a discharge NIHSS of 1 .

\section{Discussion}

Transient ischemic attacks (TIA's) and ischemic strokes secondary to aneurysm-to-artery embolism are uncommon events. In one retrospective series of 463 patients presenting with unruptured aneurysms, $2.4 \%$ of patients presented with ischemic lesions with $1.1 \%$ demonstrating partial thrombosis of the aneurysm at time of investigation ${ }^{1}$. In other case series the incidence of spontaneous intra-aneurysmal thrombosis in patients with giant intracranial aneurysms was reported as $55 \%^{2}$ with the incidence of ischemic events attributable to aneurysmal emboli as high as $7.2 \%$ in aneurysms smaller than $1 \mathrm{~cm}$ and $3.3 \%$ in aneurysms of all sizes ${ }^{3,4}$. Most ischemic events attributable to aneurysmal emboli are TIA's and the pathophysiology relates to either (a) parent artery occlusion due to local extension of the luminal thrombus (b) distal embolic occlusion secondary to dislodgement of intra-aneurysmal thrombus or (c) local effects secondary to increased mass effect ${ }^{5}$. Symptomatic CCA most commonly present with diplopia and pain, however $2 \%$ of untreated CCA may present with cerebral ischemia secondary to aneurysm-to-artery embolism ${ }^{6}$. In contrast with aneurysms within the subarachnoid space, CCA rarely cause subarachnoid hemorrhage with a reported incidence rate of $0.19 \%$ per year ${ }^{7}$.

This case represents a rare presentation of acute ischemic stroke secondary to distal embolus from an unruptured partially thrombosed CCA. European and North American guidelines regard the presence of any aneurysm to be an absolute contraindication for thrombolytic therapy. Consequently, due to the relative rarity of aneurysm to artery emboli and existing guidelines governing administration of IV-tPA scant data is available on the safety of systemic thrombolysis in ischemic events secondary to aneurysm-to-artery embolism. A literature review identified seven patients treated with thrombolytics in the setting of acute stroke and presence of unruptured and untreated aneurysms. Two cases were treated with IV-tPA without adverse events $^{8}$. Four cases were treated with intra-arterial (IA) urokinase with one fatal aneurysmal rupture, one case was treated with IA-tPA and subsequently had a fatal subarachnoid hemorrhage ${ }^{8}$. Here we describe a case of acute stroke in the setting of a partially thrombosed CCA. In this case IVthrombolysis with rtPA was administered without any adverse events. To our knowledge this is the only reported case of IVthrombolysis in the setting of presumed CCA to artery emboli. We suggest that for patients with aneurysms and large ischemic penumbra thrombolytic therapy with IV-tPA should be considered on a case-by-case basis and benefits of IV-tPA should

From the Department of Medicine, Division of Neurology, Queen's University, Kingston General Hospital, Kingston, Ontario, Canada.

Received August 3, 2010. Final Revisions Submitted September 29, 2010. Correspondence to: Jamsheed. A. Desai, Queen's University, Department of Medicine, Division of Neurology, Kingston General Hospital. Connell 7-76 Stuart Street, Kingston, Ontario, K7L 2V7, Canada. 

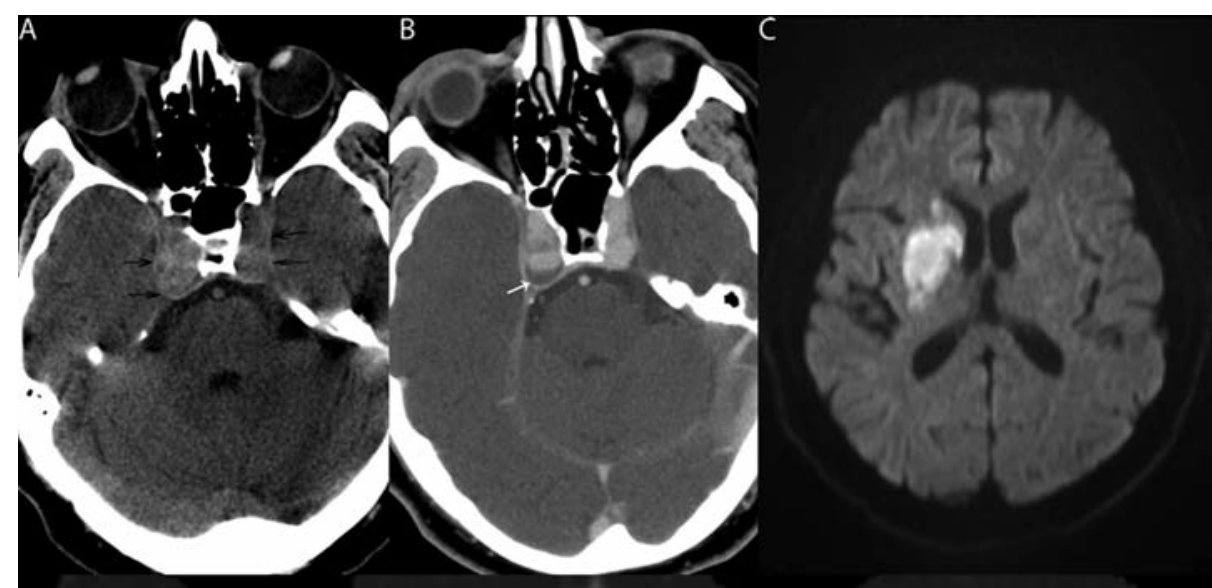

D

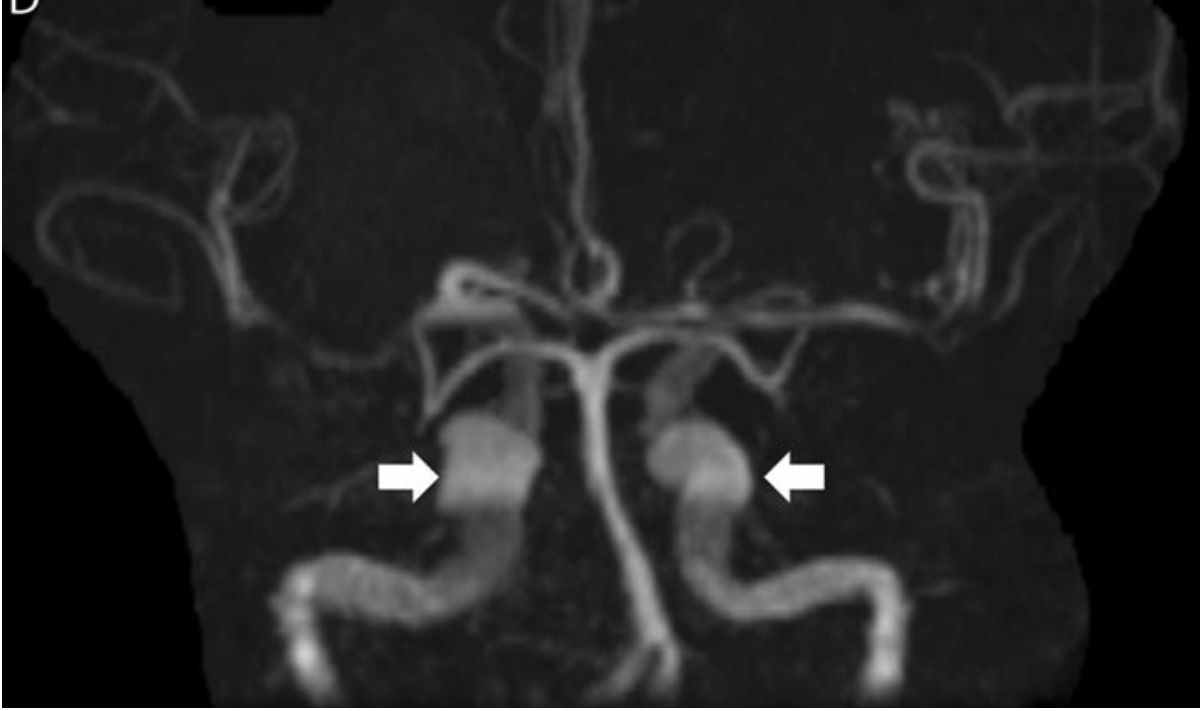

Figure: Pre-thrombolysis CT head with and without contrast and post-thrombolysis MRI. A) Axial unenhanced CT demonstrating bilateral prominence of the cavernous sinuses larger on the right [black arrows]. B) Contrast enhanced angiogram demonstrating wall irregularity and eccentric mural thrombus [white arrow] in a right carotid cavernous aneurysm. C) Diffusion weighted MRI post-rtPA outlining acute infarct in the right MCA lenticulostriate territory. D) Three dimensional reconstruction from time of flight MR angiogram demonstrating bilateral, right larger than left fusiform cavernous carotid aneurysms. be juxtaposed against risk of aneurysmal rupture. The benefit of IV-tPA in the setting of acute stroke up to 4.5 hours is clearly established ${ }^{9}$. The natural history of CCA suggests that, when confined to the cavernous sinus with no protrusions into the subarachnoid space, the propensity for spontaneous rupture is extremely low ${ }^{6,7}$. Limited data extrapolated from case series suggests that aneurysmal rupture is more likely when intraarterial rather than intravenous fibrinolytics are used but meaningful conclusions are limited by small patient numbers. An ongoing registry of patients given rtPA in the setting of treated, un-treated or newly discovered intracranial aneurysms may assist in better defining the true risk of aneurysmal rupture in this unique subset of stroke patients.

\section{REFERENCES}

1. McLaughlin N, Bojanowski MW. Unruptured cerebral aneurysms presenting with ischemic events. Can J Neurol Sci. 2008 Nov;35: 588-92.

2. Whittle IR, Dorsch NW, Besser M. Spontaneous thrombosis in giant intracranial aneurysms. J Neurol Neurosurg Psychiatry. 1982 Nov;45:1040-7.
3. Friedman JA, Piepgras DG, Pichelmann MA, Hansen KK, Brown RD, Jr., Wiebers DO. Small cerebral aneurysms presenting with symptoms other than rupture. Neurology. 2001 Oct 9;57: 1212-16.

4. Qureshi AI, Mohammad Y, Yahia AM, et al. Ischemic events associated with unruptured intracranial aneurysms: multicenter clinical study and review of the literature. Neurosurgery. 2000 Feb;46:282-9.

5. Cohen JE, Itshayek E, Gomori JM, et al. Spontaneous thrombosis of cerebral aneurysms presenting with ischemic stroke. J Neurol Sci. 2007 Mar 15;254:95-8.

6. Stiebel-Kalish H, Kalish Y, Bar-On RH, et al. Presentation, natural history, and management of carotid cavernous aneurysms. Neurosurgery. 2005 Nov;57:850-7.

7. Kupersmith MJ, Stiebel-Kalish H, Huna-Baron R, et al. Cavernous carotid aneurysms rarely cause subarachnoid hemorrhage or major neurologic morbidity. J Stroke Cerebrovasc Dis. 2002 Jan; 11:9-14.

8. Kane I, Sandercock P, Thomas B. Can patients with unruptured intracranial aneurysms be treated with thrombolysis? Cerebrovasc Dis. 2005;20:51-2.

9. Lees KR, Bluhmki E, von KR, et al. Time to treatment with intravenous alteplase and outcome in stroke: an updated pooled analysis of ECASS, ATLANTIS, NINDS, and EPITHET trials. Lancet. 2010 May 15;375:1695-703. 